\title{
DEVELOPABLE RULED SURFACES FROM A CYLINDRICAL HELIX AND THEIR APPLICATIONS AS ARCHITECTURAL SURFACES
}

Andrés Martín-Pastor, Universidad de Sevilla, Spain (archiamp@us.es)

Alicia López-Martínez, Universidad de Sevilla, Spain.

\begin{abstract}
The study of developable surfaces has not been very common in the context of architectural education. This is not due to their complexity, but perhaps to the relatively recent emergence of digital tools that enable these surfaces to be controlled via advanced graphic thinking. In our recent workshops on Geometry and Digital Fabrication, we have worked with developable helical surfaces. These workshops have involved the design, manufacture and assembly of two ephemeral pavilions: the Butterfly Gallery and the Molusco Pavilion. These two experimental structures provide the initial inspiration to enter into greater geometrical depth of the expandable condition of certain helical structures described herein.
\end{abstract}

KEYWORDS: DEVELOPABLE SURFACES, HELICOID, DIGITAL FABRICATION, EPHEMERAL ARCHITECTURE

\section{DEVELOPABLE HELICAL SURFACES}

The study of developable surfaces is strongly marked by the mathematical advances of the seventeenth century and by the figure of Gaspard Monge [1] who devoted much of his work to studying them in depth. It can be seen that the advance in knowledge of these surfaces is closely related to spatial intuition and the treatment of space as defined by Monge through his Géométrie Descriptive [2].

However, according to Glaeser [3], the presence of such surfaces is not yet common in the context of architectural education and professional practice. This is not due to their 
complexity, but perhaps to the relatively recent emergence of digital tools that enable these surfaces to be controlled via advanced graphic thinking.

Specifically, the helicoid is extensively studied in classic manuals of Descriptive Geometry, and its formulation has been described as a surface of equal slope resting on a cylindrical helix [4], [5], [6], [7]. We make a description centred on the more general conception of developable surfaces, whereby we consider it as a type of tangential surface with respect to a space curve (Fig.1) [8]. These tangential surfaces are determined as the envelope surface of the osculating planes [9] (defined by the tangent vector $T$ and the normal vector $N$ of each point of the space curve, the so-called the edge of regression). It is common to observe a simplified definition that determines this surface generated, not by the envelope of osculating planes, but directly by the set of tangent vectors $T$ along the edge of regression, as mentioned by Glaeser [3, p.63]. According to this definition, and within the general theory of surfaces, the developable surface from a cylindrical helix can be understood as a tangential developable (i.e. torsal) ruled surface of this curve.

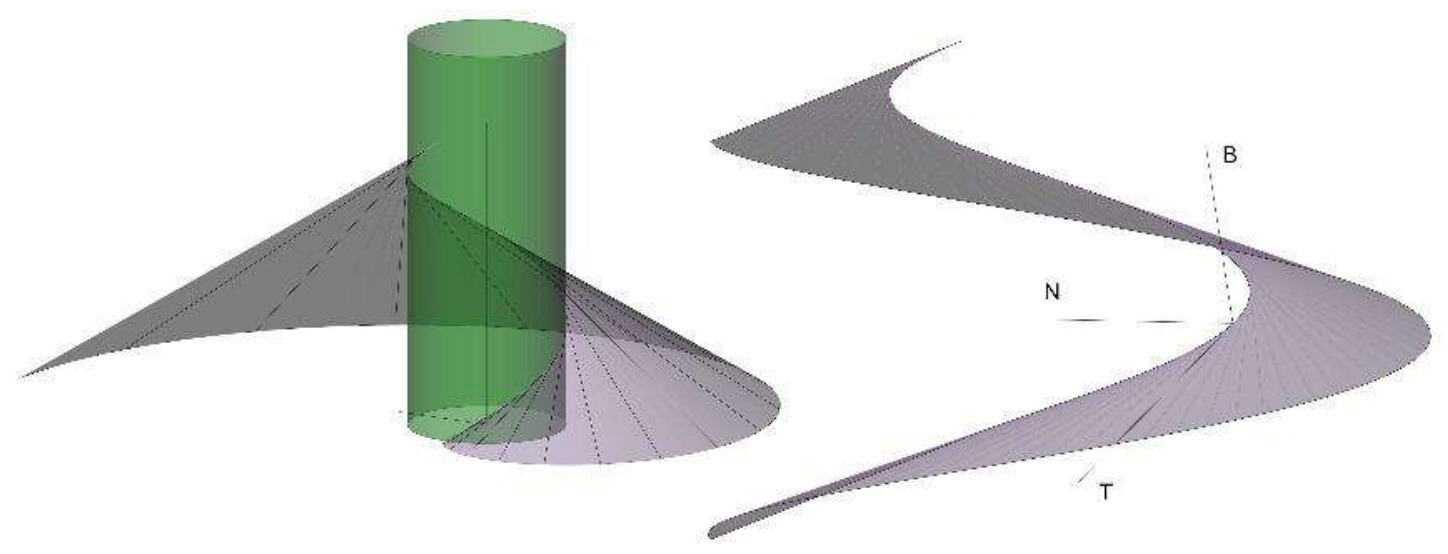

Fig. 1 - Left-hand-side: helicoid as a surface of equal slope supported on a helix. Right-hand-side: tangential developable surface generated by tangent vectors $\mathrm{T}$ along a curve. Source: The authors.

\section{Evolute, involute and evolvent}

On the other hand, we can understand the helicoid as a surface of equal slope that rests on a flat horizontal curve called involute or 'evolvent' (according to Spanish notation). These concepts are reviewed here.

In general, the evolute is the curve formed by the orbit of the centres of all osculating circles of another curve called the involute. A single evolute has infinitely many equidistant involutes (Fig. 2, left-hand-side) and the shape of the evolvent is identical for all circumferences, 
according to Elizalde [9, p.334]. This may seem contradictory to the aforementioned property: a single evolute (circumference) has infinite involutes (evolvents), but as shown in Fig. 2 (left-hand-side), the infinite evolvents would be interpreted as the different rotations of the curve around the centre of the circumference. The turns produce equidistant involutes and these become into the original in a complete rotation. Hence the division of the circumference into $n$ equal parts, is directly related to the division of segment $A-A^{\prime}$ (tangent to the circumference) into the same $n$ equal parts. The evolvent also maintains another important property with respect to its circumference: The distance between the start point $A$ and the end of its first rotation $A^{\prime}$ is strictly $2 \pi R$, the length of the circumference. This relationship can be extrapolated to any proportional distance (Fig. 2, centre).
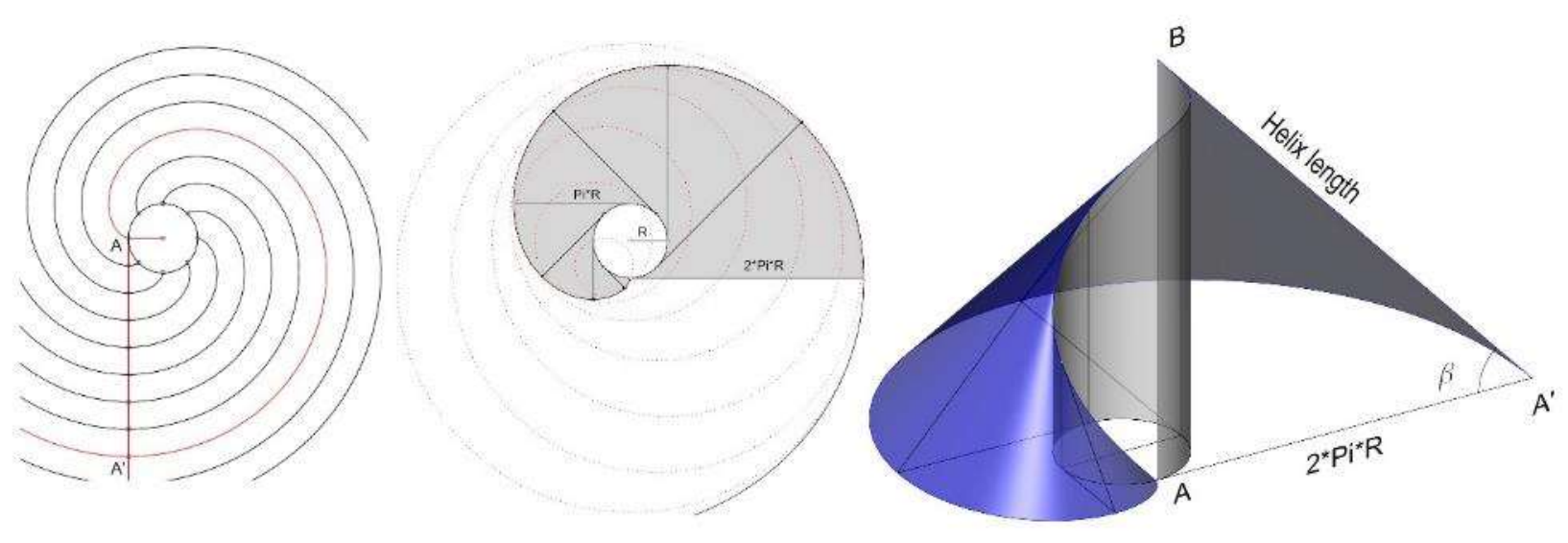

Fig. 2 - Left-hand-side: Infinite evolvents of the circumference.

Centre: relationship between the length of the circumference and its evolvent.

Right-hand-side: Graphic demonstration. Source: The authors.

The helix has a constant slope $\beta$, just like the straight lines of the helicoid. If we unroll the vertical cylinder that carries the helix $A-B$, it becomes a segment $A^{\prime}-B$, since that cylinder is the rectifying surface of the helix [8, p.72]. The rectified segment $A^{\prime}-B$ must be the length of the helix and the segment $A-A^{\prime}$ must be the length of the circumference. We can graphically verify this spatial correspondence between the unrolled cylinder and the helicoid (Fig. 2, right-hand-side). The rectified segment $A^{\prime}-B$ coincides with the generating lines of the helicoid and the evolvent passes through point $A^{\prime}$.

\section{FACTORS OF THE DEVELOPMENT}

\section{Development by circular sectors}


If the helicoid is formed by generating lines of equal length, as in Fig. 3, then its planar development is a portion of a circular sector limited by two lines tangent to the smaller circumference [9, p.338]. The length of the arc of this smaller circumference is equivalent to the length of the cylindrical helix on which the straight lines are tangentially supported.

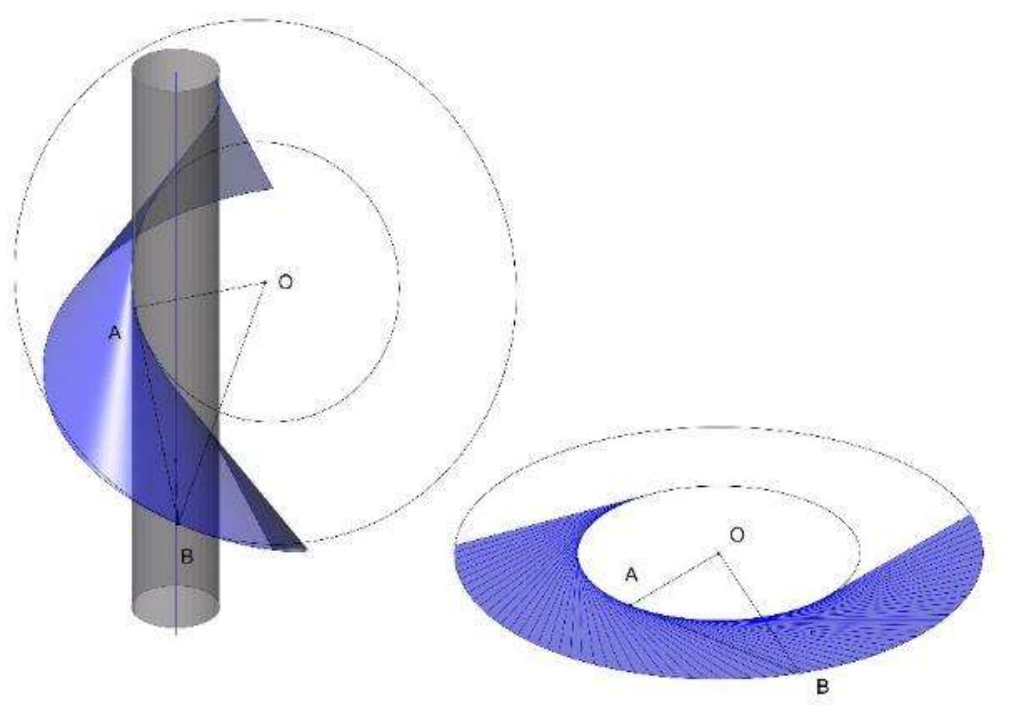

Fig. 3 - Relationship between the osculating circle in space and the planar development. Source: The authors.

The curvature at each point of the generating helix is defined by the osculating circle, and the radius of curvature $O A$ coincides with the radius of the minor circumference in the development. The points $O B$, of the right-hand-side triangle contained in the osculating plane passing through point $A$, determines the largest circumference (Fig. 3). We can imagine how the planar development curves in space until it attains the helicoidal form (Fig. 3 left-hand-side). Therefore, the same planar development could be considered common to several helicoids.

\section{Developments of helicoids generated by evolvents}

If the helicoid is generated as an equal slope surface that rests on an evolvent, then it can be verified that the unrolled surface is another evolvent (Fig. 4). The first evolvent comes from the circle projection of the helix, and the second from the osculating circle of the helix. The most important property, which we will use later, is that both evolvents, and their respective circles, have a homothetic relationship. 

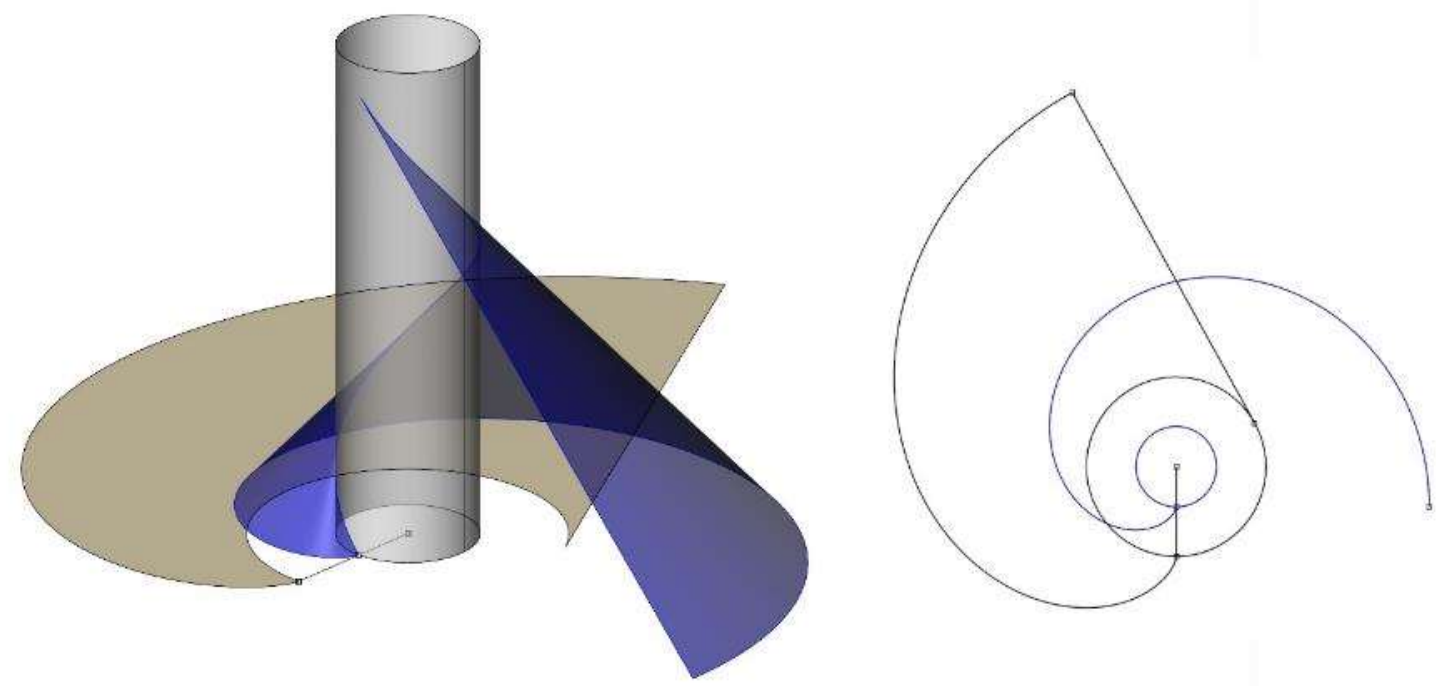

Fig. 4 - Left-hand-side: Relationship between the evolvent of the basis and the unrolled helicoid. Right-hand-side: Homothetic relation between both evolvents. Source: The authors.

\section{CURVATURE OF A CURVE AND CURVATURE OF A SURFACE}

The main curvature $\delta$ of a curve (or simply, curvature) measures the angular deviation between two normal vectors $N$, infinitely close at a point on the curve. This curvature is characterized graphically by the osculating circle, which is defined by three points of the curve that are infinitely close to each other.

In contrast, the torsion $\zeta$ of a curve evaluates the tendency of this curve to rise from its osculating plane and it is measured by the angular deviation between two Binormal vectors $B$, that are infinitely close. Once the curve unfolds on a plane, it maintains the main curvature but it loses the torsion, according to Leroy [8, p. 143].

The evolution of the values of $\delta$ and $\zeta$ can be verified by their equations, by means of substituting values:

$$
\begin{aligned}
& \text { Curvature } \delta=\frac{R}{R^{2}+K^{2}} \quad \text { Torsión: } \zeta=\frac{K}{R^{2}+K^{2}} \\
& x(t)=R \cos t \quad y(t)=R \sin t \quad x(t)=K t
\end{aligned}
$$

The curvature of a surface, in general terms, is characterized by two principal curvatures determined by two principal directions $\mathrm{V}, \mathrm{U}$. In the case of the developable surfaces, one of these two curvatures is always zero, since it coincides with the direction of the generating lines. For this reason, we usually denominate the developable surfaces as surfaces of a single curvature, which is equivalent to having a vanishing Gaussian curvature, or as formed only by parabolic points (see Glaeser [3, p.61]). 
This has major practical consequences; the curvature of these surfaces is characterized in each point by a single parameter of curvature associated perpendicularly to the direction of the generating lines. It can therefore be expressed graphically as a circle of curvature, as seen in Fig. 5.

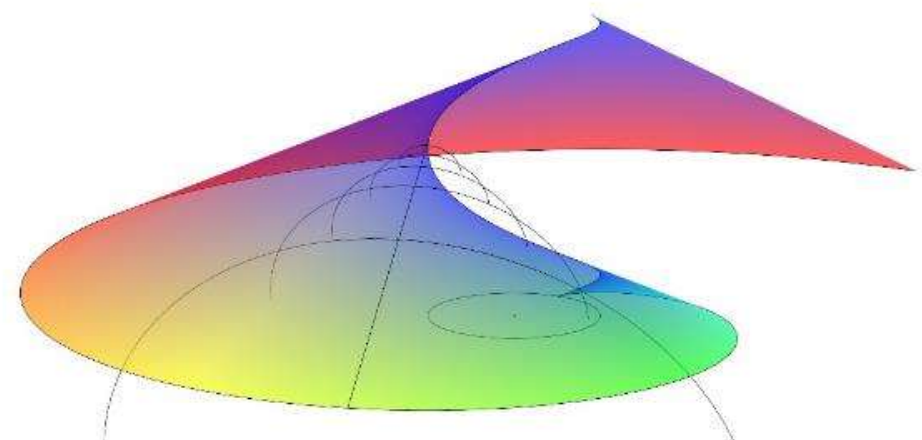

Fig. 5 - Curvature of a developable surface characterized at each point by unique parameters (circle of curvature) perpendicular to each generating line. Source: The authors.

If we relate these geometrical aspects to the possibility of constructing these surfaces with laminar materials, such as wood, veneers, etc., then this curvature value can help us determine the feasibility of using such materials. The appropriate use of these materials would be limited between an infinite radius of curvature (zero curvature) and a minimum radius (maximum curvature of the material) [10]. To conclude this first approach to design, we use another type of analysis where tensions and deformations are considered.

\section{HELICOIDS THAT SHARE THE SAME DEVELOPMENT}

Once the geometrical foundations are reviewed, we establish that several helicoids can share the same planar development, since this property explains why they can be turned into transformable structures.

We have formulated a parametric algorithm - based on tangents $T$ along the regression edge - employing Rhinoceros-Grasshopper software. We have been able to relate how the flat development of a helicoid varies by changing, or keeping constant, different parameters (radius $\mathrm{R}$ of the helix, pitch of the helix, radius of the osculating circle,...) as we will show below. 


\subsection{Helicoids that share the same planar development}
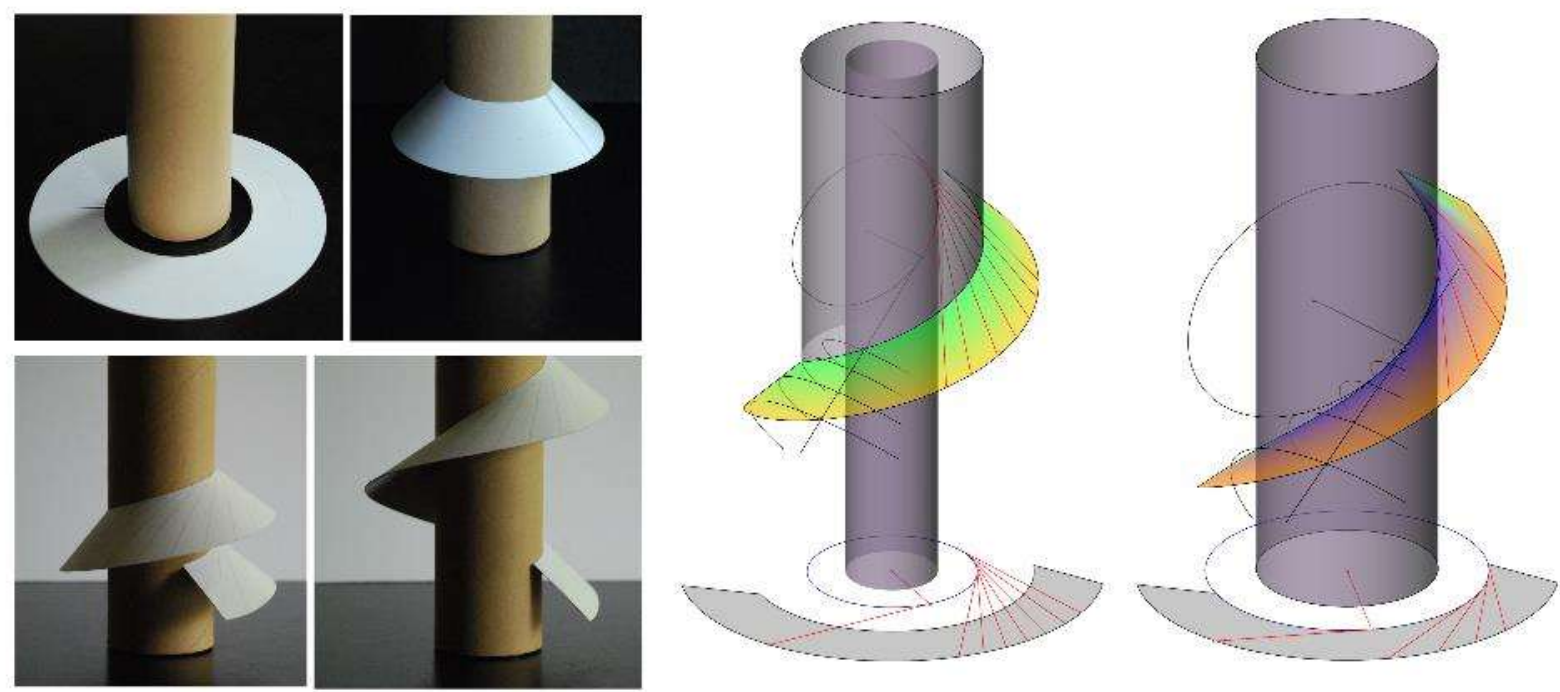

Fig. 6 - Left-hand-side: Different helicoids with similar development, around the same cylinder.

Right-hand-side: Evolution of the generating lines to maximum position (right-hand-side figure). The maximum curvature occurs in the proximity of the helix, where the lines are tangent. Source: The authors.

The same planar development offers an infinite number of possible helicoids since, in fact, there are two parameters to combine. First, the radius $R c$ of the cylinder is limited between zero and the smallest circle of the development. Secondly, we can vary the pitch of the helix for each $R c$ chosen. The pitch would be comprised between two limits: zero, at which the helicoid acquires the form of a cone coiled on the cylinder; and a maximum pitch position for each Rc.

In Fig. 6 (left-hand-side), it can be observed how the planar development can be adjusted around the same cardboard cylinder $R 1$ in different helicoids, varying from zero pitch (cone), to the maximum pitch position. In the graphic study of Fig. 6 (right-hand-side) we verify that the generating lines of the helicoid are tangent to another invisible inner cylinder, which, in the case of maximum pitch, coincides with the cylinder of radius $R 1$.

\subsection{Family of helicoids that share the same development (circular sector) and whose} generating lines are tangent to the helix

In order to study how a series of helicoids share the same development, the length of the helix and the radius of the osculating circle are kept constant, while the radius of the helix is varied (Fig. 7). This radius thus changes from the maximum value (coincident with the radius of the osculating circle in planar position) to a value close to zero. As the radius of the helix is reduced, it also decreases the pitch of the helix. The helix must maintain the same length; consequently, the helicoid begins to roll up. In the limit position (radius close to zero) the 
helix tends to become a vertical segment, since it has been coiled almost an infinite number of times.

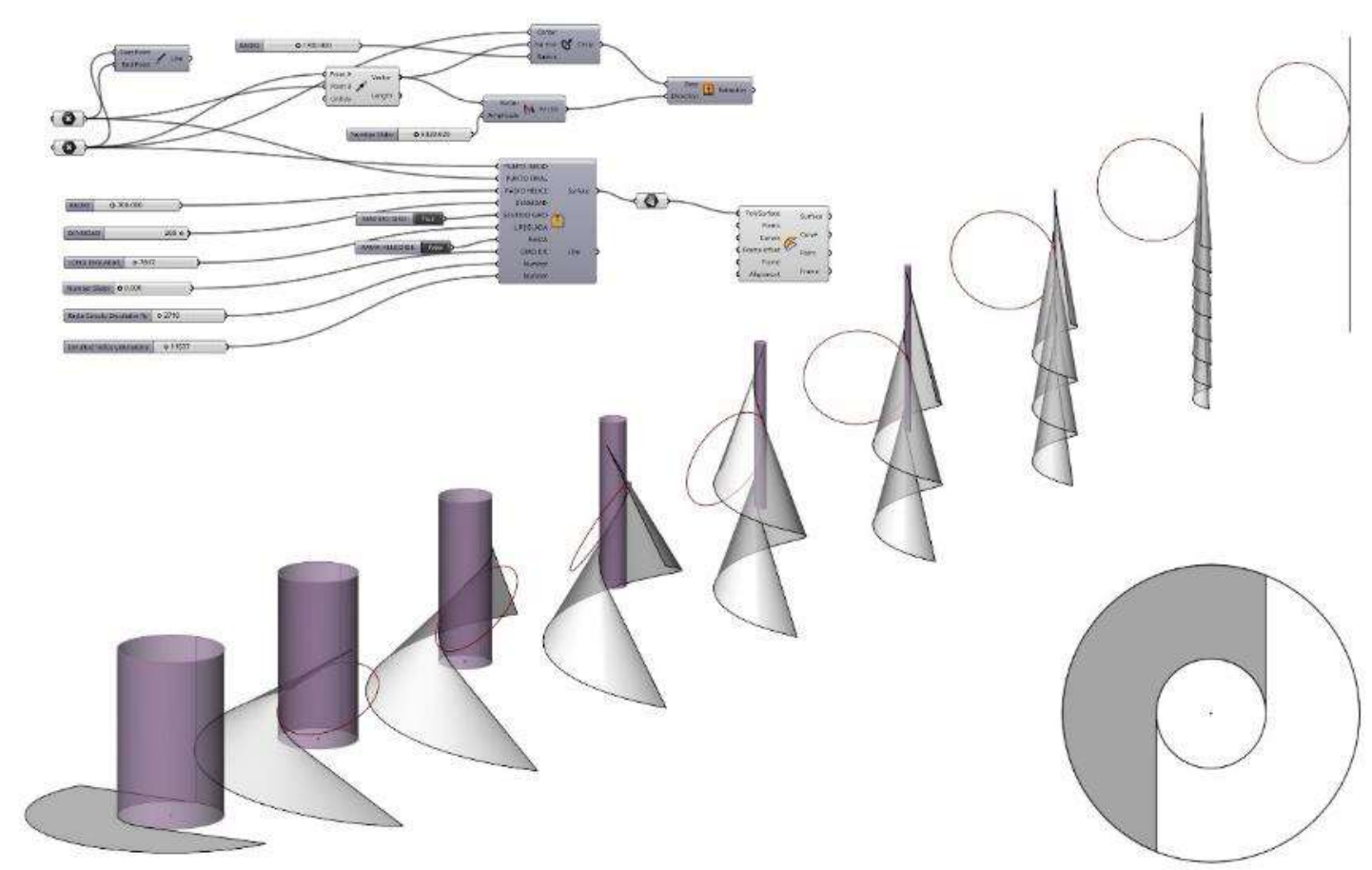

Fig. 7 - Family of helicoids that share the same planar development and whose generating lines are tangent to an interior helix of variable radius. It can be appreciated how all the generating helices share the same osculating circle radius. Source: The authors.

\subsection{Development by evolvents}

Similarly, when the helicoids are generated by evolvents, the same planar development is common to several helicoids, which guarantees its transformable character as shown in Fig. 8. It can be observed how the radius of the helices decreases homothetically with the evolvent (Fig. 8, top). From another point of view, we can deduce that all the helicoids supported by the same evolvent, regardless of the slope of the helical surface have, as edge of regression, helixes of equal radius $R$ (Fig. 8 , down-left). So, we conclude that we can always create a transformable structure from any two helicoidal developments, joining the evolvents and providing that both curves are of equal length.

The two helicoids that share the evolvent, will have two generating helixes of equal radius at each position (Fig. 8, down-cent From another point of view, it can be deduced that all the helicoids supported by the same evolvent, regardless of the slope of the helical surface, have helices of equal radius $R$ as the edge of regression (Fig. 8, bottom left-hand-side). It can therefore be concluded that we can always create a transformable structure from any two helicoidal developments, if we join the evolvents on the condition that both curves are 
of equal length. The two helicoids that share the evolvent will have two generating helices of equal radius at each position (Fig. 8 , bottom centre).

Fig. 8 - Top: Family of helicoids that share the same planar development and whose generating lines are tangent to helices. Bottom left-hand-side: Two helicoids supporting the same evolvent always share the radii of their generating helixes. Source: The authors.
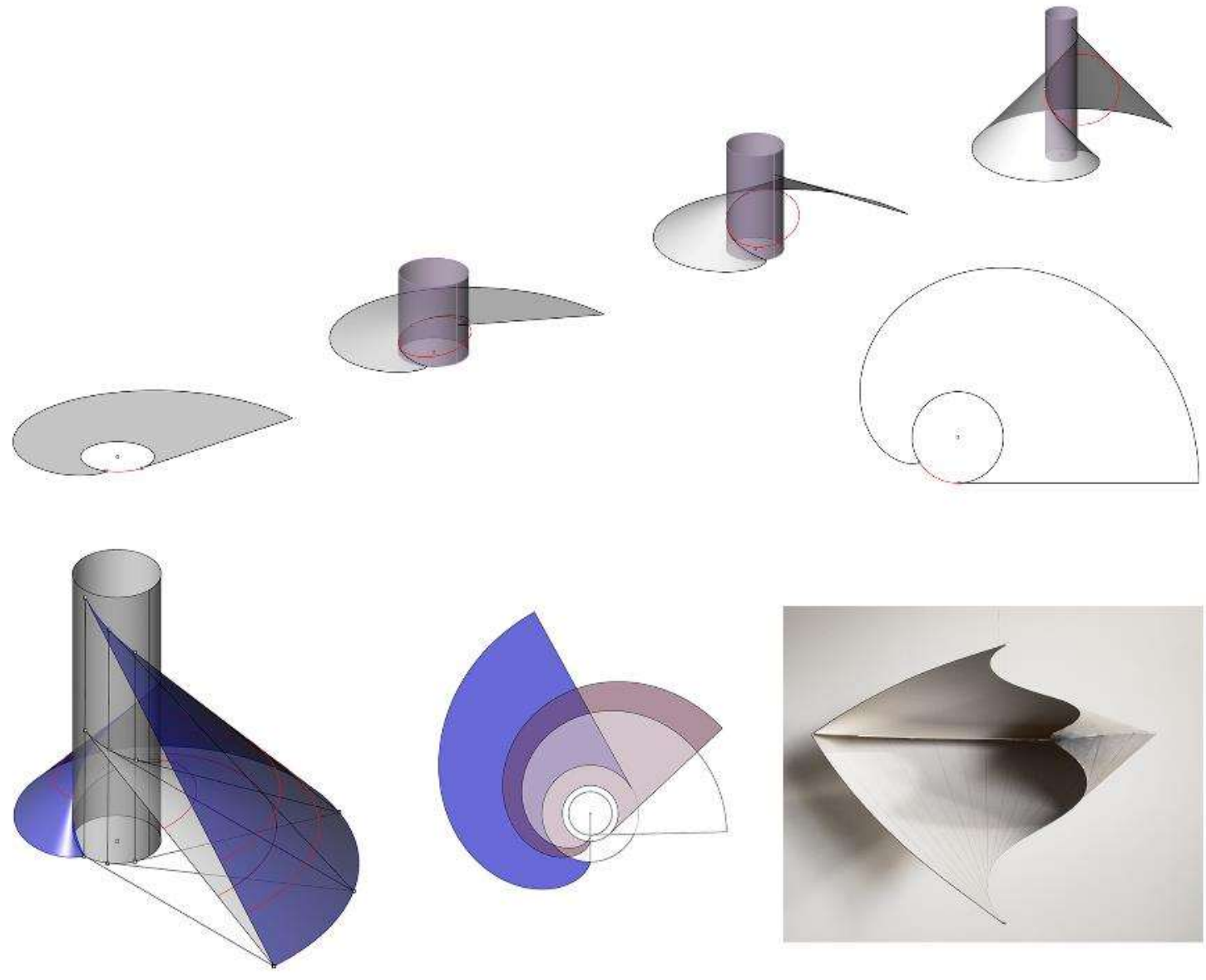

A unique position of this transformable structure formed by two helicoids occurs when one of them is plane, denoting the minimum angle of the other helicoid. If a third helicoid is added, then we arrive at one formal solution of great structural resistance (Fig. 8, bottom right-hand-side). As Fig.8 demonstrates, in addition to the explanation, the helicoids that share the same evolvent are related to a spatial affinity transformation.

\section{TRANSFORMABLE HELICOIDAL FOLDED STRUCTURES}


We now propose a set of transformable helicoids, formed by plane surfaces sewn at their edges.

\subsection{Folding on concentric rings}

We propose a series of circular sectors with their edges sewn at their contact line, which allows the movement of the whole set. We will consider the physical movement involved in carrying the folded geometric structure from the plane to space (Fig. 9).
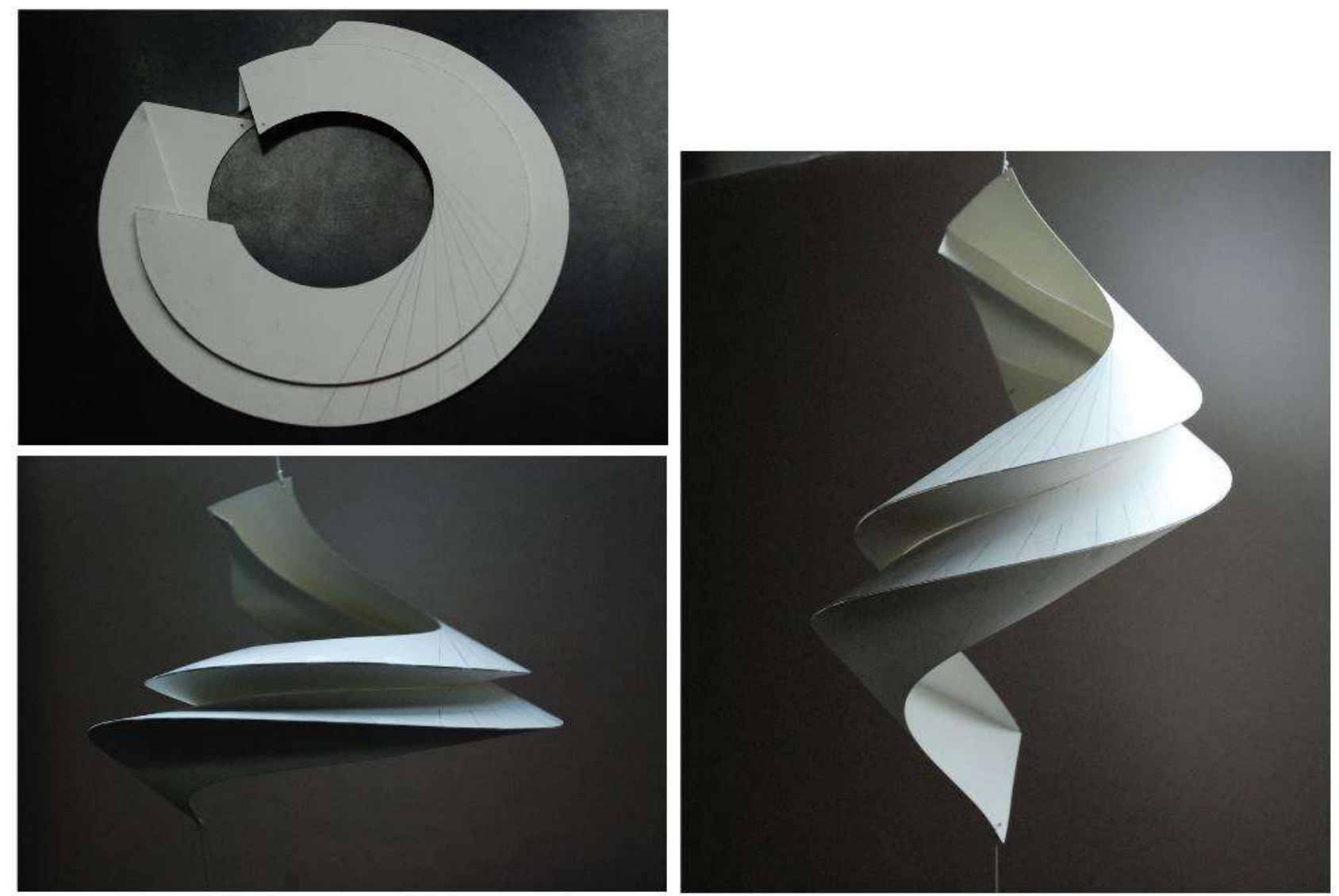

Fig. 9 - Set of circular sectors, connected at their common edge.

Evolution from their folded and plane position to varius helical rings. Source: The authors.

We created a model to study different positions in its extensible movement and to clarify which geometric relationships link the helicoid family with the motion of the set (Fig. 9). It is first deduced that all the rings of the set are helicoids of equal pitch. It is important to highlight how contact occurs between generating lines and their respective apparent helices. Only a truly tangential contact occurs in the case of the smaller rings, the rest of the ruled lines of the set are not tangent to the apparent helices, but to other inner helices (Fig.10). These helices have a radius similar to the smallest circumference of the circular sector. It can therefore be deduced that the whole set depends on a unique generator helix, and all helical rings are fragments of one or the other branch of the same helicoid in different rotations. 

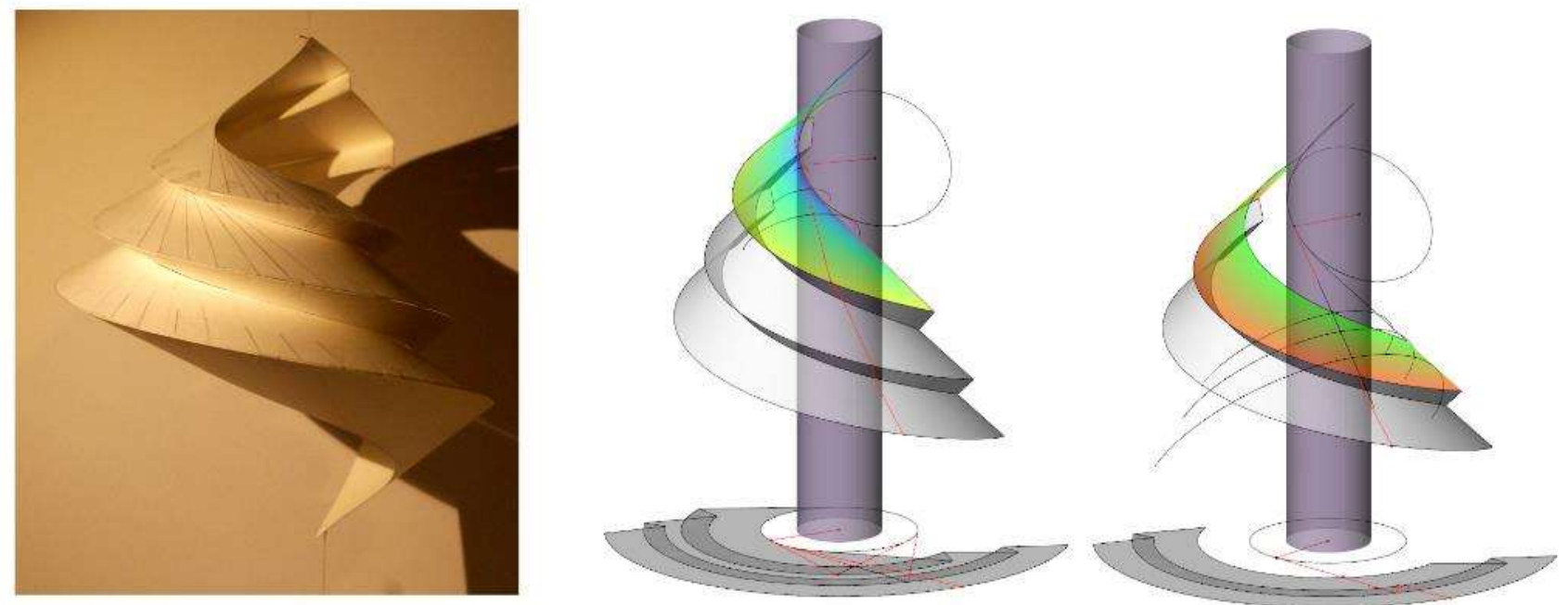

Fig. 10 - Left-hand-side: Structure formed by a group of helical rings. Centre and right-hand-side: The ruled lines of the second ring are tangents to the cylinder of the minor ring. The maximum curvature of surface occurs in the proximity of the minor ring helix. Source: The authors.

As we have already observed, the generating lines of the helicoid are tangent to the helix only when the maximum pitch position is reached, and hence the helicoid corresponding to the minor ring is always in the maximum pitch position (Fig.10, centre). If this parameter (pitch) is kept invariant, then we diminish the degree of freedom and the expandable structure has only one possible transformation path. With more tension exerted, and the maximum pitch mode achieved, the smaller helicoid is deformed only by vertical lengthening, while its radius is reduced. In this way, in its motion, the whole transformable structure depends solely on the smaller ring.

\subsection{Folding evolvents.}

Another possibility of folding consists of articulating the helicoids with respect to a mirror plane, which imposes the condition of equal slope to that plane. Since the helicoid is a surface generated by a family of ruled lines of equal slope with respect to the plane of the evolvent, the set of lines could be reflected specularly from this plane, thereby retaining the same slope in both cases (Fig.11). The evolvent can, therefore, be understood as the folding line of a single continuous surface (Fig.11 bottom). 


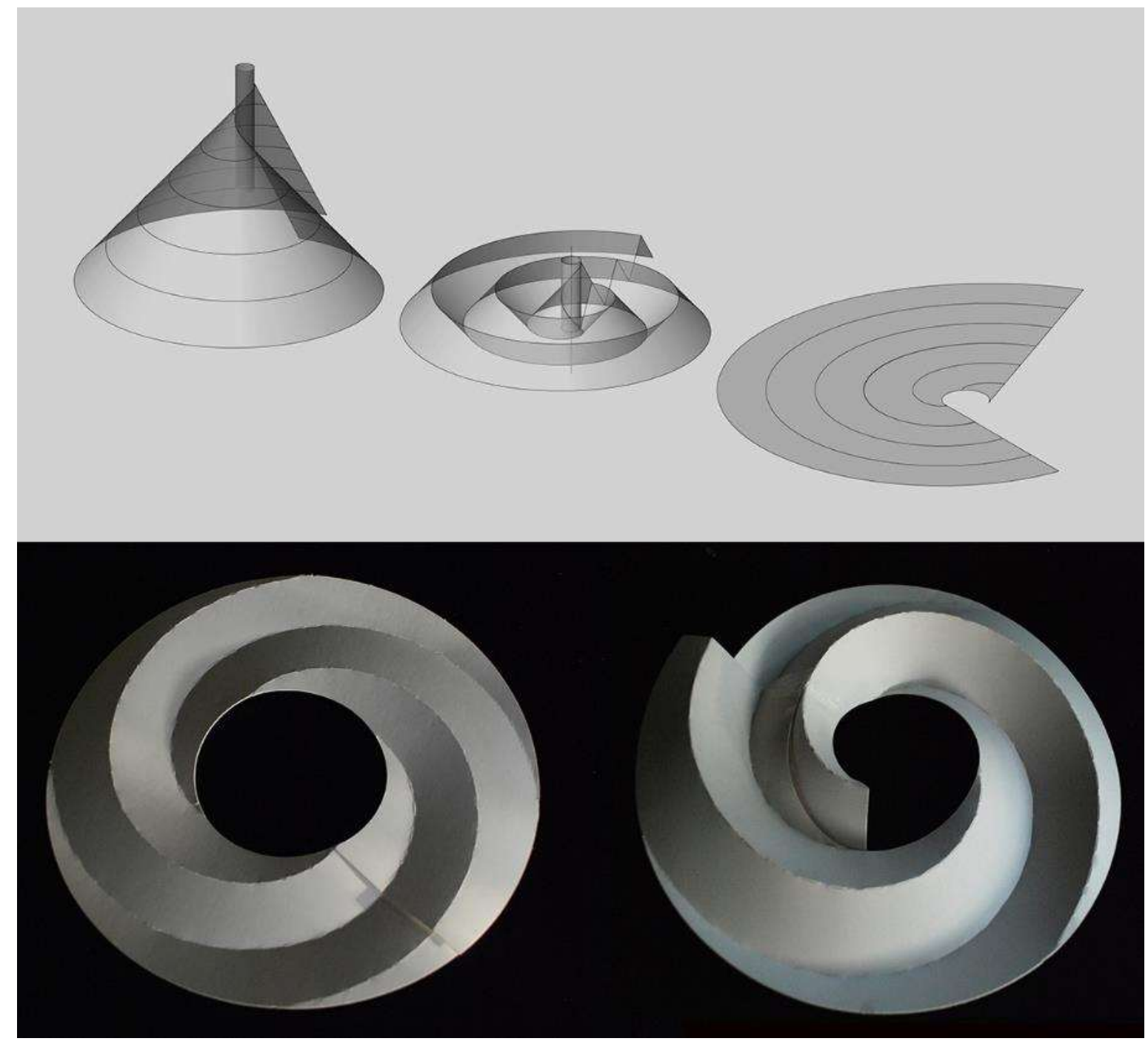

Fig. 11 - Top: Sections (evolvents) of a helicoid using horizontal planes.

Bottom: A single piece of cardboard folded along evolvents. Source: The authors.

\section{CONCLUSIONS AND DISCUSSION}

We have analysed the generation of helicoids from their parameterization in RhinocerosGrasshopper, and have paid special attention to developments in circular rings and evolvents. We are now able to propose structures formed by several helical surfaces linked together, giving rise to a single transformable or extensible structure. These surfaces can be built with laminar pieces, which can be combined with different folded structures, thereby providing a wide variety of formal solutions. We have studied the geometric relationships between the helicoids in the movement of transformation of a linked set, and have demonstrated the importance of certain geometric parameters over others. All this expertise and knowledge can be put into practice when we design helical surfaces for use in lightweight architecture. At this point, we have designed two architectural installations on the 
general properties of the helicoids (Figs. 12 and 13), [11]. The next challenge is now to design an extensible structure that will benefit from the characteristics studied.

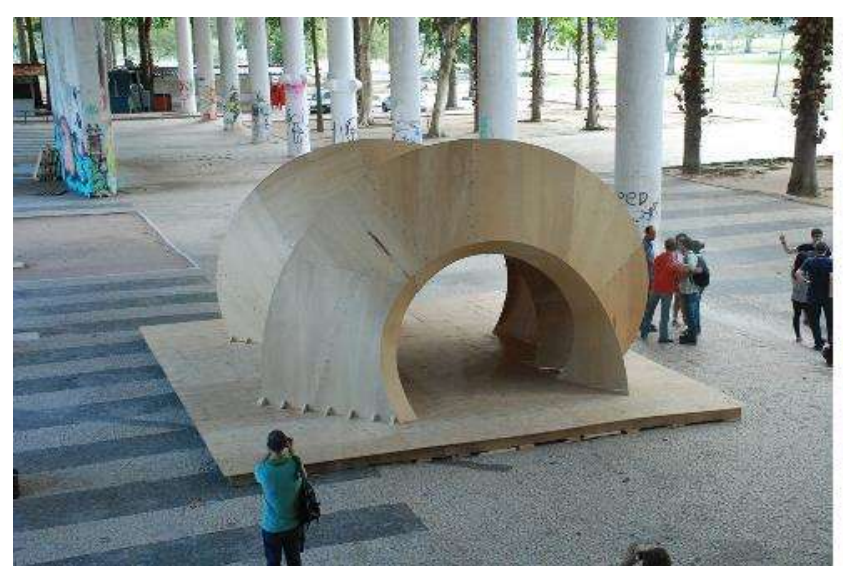

Fig. 12 - The Butterfly Gallery, Universidade Federal do Rio de Janeiro, Brazil, 4-14 August 2015. Source: The authors.

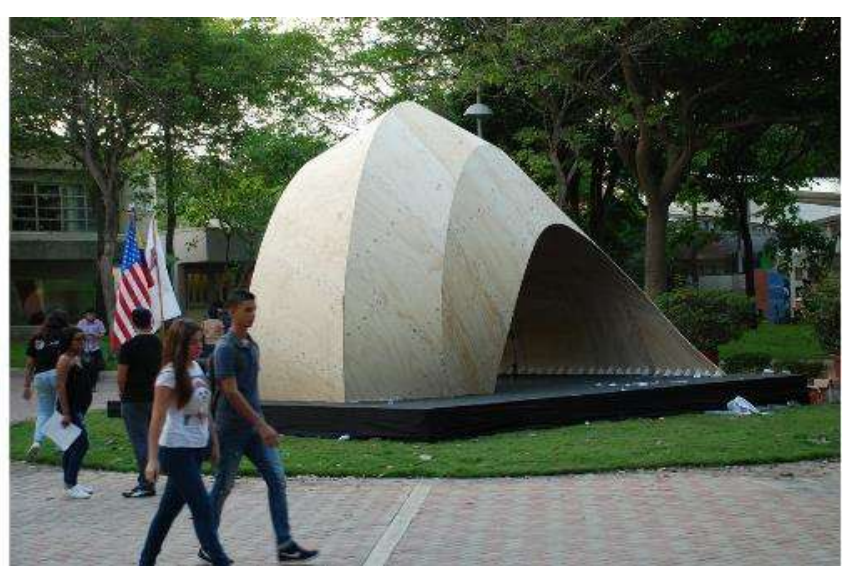

Fig. 13 - The Molusco Pavilion. Facultad de Arquitectura, Urbanismo y Diseño. Universidad del Norte, Barranquilla, Colombia. October 2016. Source: The authors.

\section{BIBLIOGRAPHIC REFERENCES}

[1] Monge. G. (1769). Sur les développées des courbes à double courbure et leurs inflexions. Journal encyclopédique: 284-287.

- 1780. Mémoire sur les propriétés de plusieurs genres de surfaces courbes, particulièrtement sur celles des surfaces d'eveloppables, avec une application à la theorie des ombres et des pénombres. Mémoires de divers sçavans 9, 9: 593-624 (written 1775).

- 1785. Mémoire sur les développées, les rayons de courbure, et les différents genres d'inflexions des courbes à double courbure. Mémoires de divers sçavans, 1, 10: 511-50 (written 1771).

- 1795a. Feuilles d'analyse appliquée à la géométrie. Paris. (Second ed. with the title e Applications de l'analyse à la géométrie. Paris: Baudouin, 1811).

- 1795b. Géométrie Descriptive. First ed. in e Les Séances des écoles normales recueillies par des sténographes et revues par des professeurs, Paris.

[2] Lawrence, S. (2011) Developable Surfaces: Their History and Application. Nexus Network Journal, 13(3). 701-714. doi: 10.1007/s00004-011-0087-z

[3] Glaeser, G. \& Gruber F. (2007). Developable surfaces in contemporary architecture. Journal of Mathematics and the Arts, 1(1). 59-71. doi: 10.1080/17513470701230004

[4] Izquierdo Asensi, F. (1986). Geometría descriptiva superior y aplicada. Madrid: Dossat.

[5] Gentil Baldrich, J.M. (1998). Método y aplicación de representación acotada y del terreno. Bellisco.

[6] Rodrigues, A. J. (1945). Geometría Descritiva [Vol. 2]. Río de Janeiro: Imprenta Nacional. 
[7] Taibo Fernández, A. (1983). Geometría descriptiva y sus aplicaciones. (Vol. 2). Madrid: Tébar Flores.

[8] Leroy, C.F.A. (1877). Traité de Geométrie Descriptive [10ª edition]. Paris: Gauthier Villars.

[9] Elizalde, J. A. (1882). Curso de Geometría Descriptiva [\& Atlas], Madrid: Manuel Tello.

[10] Narváez-Rodríguez; R., Martin-Pastor, A.; \& Aguilar-Alejandre, M. (2014). The Caterpillar Gallery: Quadric Surface Theorems, Parametric Design and Digital Fabrication. Advances in Architectural Geometry 2014, (309-322.). Springer. doi: 10.1007/978-3-319-11418-7_20

[11] Martín-Pastor, A. (2016). Um retorno aos fundamentos da geometría. The Butterfly Gallery, Estratégias Geométricas para a Fabricação Digital. Cadernos PROARQ. Revista de Arquitetura e Urbanismo, 25, 2-30. Retrieved from:

http://cadernos. proarq.fau.ufrj.br/en/paginas/edicao/25

\section{BRIEF BIOGRAPHY}

Andrés Martín-Pastor [ORCID: 0000-0002-5588-2886, ResearcherID: K-9597-2013] is an architect with a PhD from the Universidad de Sevilla (Spain), where he works as a lecturer in the Department of Graphic Engineering. His research is focused on perspective and geometry in architecture and makes a thorough review ranging from the inherited graphic tradition until today's digital tools. His research also includes the study of developable surfaces and their applications in lightweight architecture. In 2015, he received the Emporia Silver Award Innovation in Ephemeral Architecture. He has lectured at several International Universities and has published numerous books, chapters and articles.

Alicia López-Martínez holds degrees in Building Engineering and a master degree in Engineering. She is a PhD student at Universidad de Sevilla (Spain). Her main research interest lies in the application of developable surfaces and digital fabrication in architecture. 